sont en réalité de fausses diarrhées, dûes à une irritation de l'intestin, et que pourraient calmer tout aussi bien les sels de ehaux, sont remarquablement améliorées par l'emploi du lait condensé qui redonne aux selles du nourrisson l'apparence normale.

\title{
DOSAGE DU CHLORE ET DU SODIUM DANS LES LAITS DE QUELQUES FEIMELLES DE MAMIMIFËRES
}

\author{
par M. L. BARTHE, \\ Professeur de toxicologie et d'hygiène appliquée à la Faculté de médecine, \\ Correspondant national de l'Académíe de médecine \\ et M. E. DUFILHO, \\ Préparateur du cours.
}

Après les dosages du chlore et du sodium dans le lait de vache et dans les eaux minérales, qui nous ont donné des résultats nouveaux et intéressants(1) nous avons porté nos recherches sur le dosage des mêmes éléments dans différents laits de femelles de mammifères.

Nous avions déjà constaté et signalé(2) que dans un troupeau de vaches hollandaises, la moyenne du sodium était de $0 \mathrm{gr} .345$ par litre, et que dans un troupeau de vaches bordelaises, cette moyenne était de 0 gr. 445 par litre. Le chiffre normal du sodium dans le lait de vache, ne dépasse jamais 0 gr. 50 par litre (indice du sodium). Nous avons vu également que l'indice du chlore ne dépassait jamais normalement $1 \mathrm{gr} .50$ par litre, et que la comparaison de ces deux indices pouvait permettre au chimiste de conclure à la fraude du lait de vache par addition de sels de sodium.

L'étude ultérieure des laits de femmes et de juments, aux diverses périodes de lactation, - toujours au point de vue du chlore et du sodium, - nous a permis d'envisager la question à un nouveau point de vue, et nos constatations ont fait l'objet d'une communication à l'Académie des Sciences(3).

Nous présentons, ci-dessous, en deux tableaux synoptiques, les résultats analytiques obtenus avec les laits de femmes et les laits de juments ; ces laits ont tous été prélevés en présence de l'un de nous. Les laits de juments provenaient de la jumenterie "Mimosa ", à Bègles (Gironde), appartenant à M. Roudel, auquel nous sommes heureux d'adresser nos remerciements. Les juments étaient soumises au régime du vert, mais recevaient en plus, chacune et chaque jour, 2 kilos d'une provende composée de tourteaux d'arachides, de caroubes, de recoupes de fèves.

(1) C.R. Acad. des Sciences, séance du 7 juin 1926, et Bulletin de la Soc de Pharmacie de. Bordeaux, 1926, t. III, p. 162, et t. IV, p 218.

(2) Loc. cit.

(3) Communication à l'Aeadémie des Seiences Séance du 17 août 1927. 


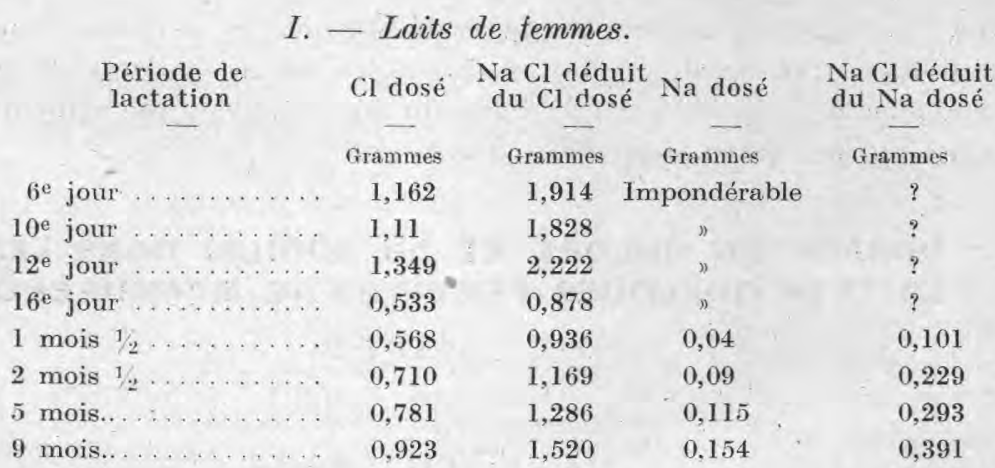

II. - Laits de juments.

a) Analyses du 26 mai 1927.

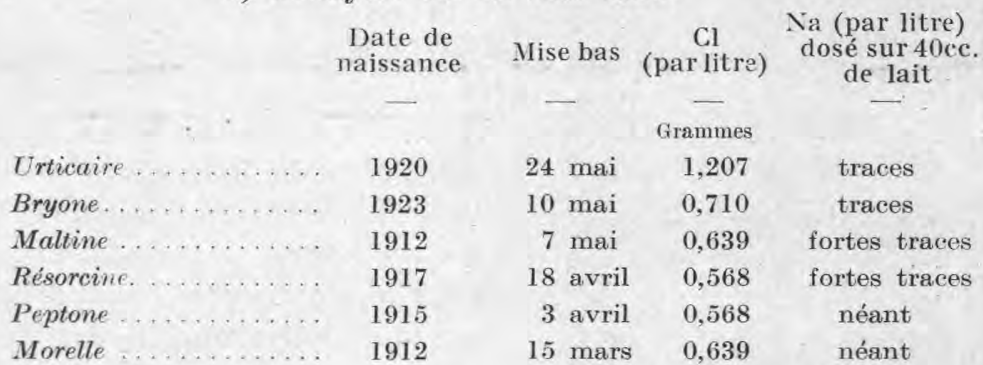

b) Analyses du 16 juin 1927 .

$\begin{array}{lccccc}\text { Maltine ............ } & 1912 & 7 \text { mai } & 0,705 & \text { traces } \\ \text { Misticouette . . . . . . } & ? & 28 \text { mai } & 0,713 & \text { traces } \\ \text { Urticaire . . . . . . . . } & 1920 & 24 \text { mai } & 0,570 & \text { treaes }\end{array}$

Pour confirmer les résultats négatifs en sodium dans le lait de juments, nous avons réuni les différents échantillons des laits précédents (soit un volume de $150 \mathrm{cc}$.$) ; nous avons évaporé le mélange, dont le résidu a été$ ensuite incinéré avec toutes les précautions nécessaires. Dans la solution acétique, après déphosphatation, le réactif Streng-Blanchetière ne nous a pas donné de précipité pondérable.

Il y a donc moins de 0 gr. 006 de sodium par litre dans le lait de jument, limite de sensibilité du réactif de Streng-Blanchetière.

Ces différents résultats nous autorisent à conclure :

Pour le lait de femme, que dans ce lait, la proportion de $\mathrm{Cl}$ est supérieure à celle du $\mathrm{Na}$, et aussi à la quantité de chlorure de sodium déduite de celle du Na dosé directement.

Par conséquent, dans le lait de femme, le chlore est compté à tort en $\mathrm{NaCl}$ : il est donc combiné à d'autres éléments, probablement au calcium, très abondant dans les laits de femme et de jument.

En période colostrale, le $\mathrm{Cl}$ dépasse la proportion de 1 gramme par 
litre, pour se fixer ensuite, pendant quelques semaines, aux environs de 0 gr. 60 à 0 gr. 70 , puis augmenter avec le $\mathrm{Na}$, quand augmente la période de lactation. Le Na n'est pondérable, dans le lait de femme, qu'à partir du $45^{\mathrm{e}}$ jour environ.

Pour le lait de jument, que, dans la période colostrale, le Cl se trouve aussi dans une proportion supérieure à 1 gramme, pour se fixer ensuite aux environs de $0 \mathrm{gr}$. 50 à $0 \mathrm{gr}$. 70 par litre. Il n'y a que des traces de $\mathrm{Na}$ à toutes les périodes de lactation, du moins pendant les premiers mois.

Ces différentes constatations rapprochent très sensiblement les laits de femme et de jument, mais les différencient du lait de vache. Elles démontrent, tout d'abord, que, jusqu'ici, le chlore dosé dans les différents milieux biologiques qui le contiennent a été exprimé à tort en $\mathrm{NaCl}$, notamment en ce qui concerne les laits.

Elles conduisent, enfin, à des considérations physiologiques très importantes - en n'envisageant toujours que les proportions de chlore et de sodium :

$1^{0}$ Il semble que le sodium ne soit pas utile à l'organisme du nouveauné, au moins dans les premières semaines de la vie, et qu'il ne serve que de support aux différents éléments nécessaires au développement de l'enfant;

$2^{\circ}$ Le lait de jument est à rapprocher du lait de femme, et peut, dans une certaine mesure, être substitué à celui-ci, comme l'ont déjà conseillé de nombreux hygiénistes;

$3^{\circ}$ A partir du cinquième mois de lactation, le lait de femme se rapproche de plus en plus du lait de vache, et si, à ce moment, la mère ne pouvait continuer à allaiter son enfant, e'est alors qu'il y aurait le moins d'inconvénients à substituer le lait de vache au lait maternel, pour l'alimentation du nourrisson.

(Travail du laboratoire de toxicologie et d'hygiène appliquée de la Faculté de médecine et de pharmacie de Bordeaux.)

\title{
LE SYNDROIVIE ATHREPSIE CHEZ LES ANIMIAUX
}

\author{
par le $\mathrm{D}^{r}$ V. BALL, \\ Professeur à l'Ecole vétérinaire de Lyon
}

Chez les animaux et notamment chez les jeunes carnivores, les troubles de la nutrition ne sont pas rares dans les premiers mois de la vie. Une alimentation défectueuse ou insuffisante est capable d'entraîner des phénomènes de dénutrition conduisant d'abord à l'hypothrepsie, puis à l'athrepsie, à l'instar de ce qu'on observe chéz l'enfant.

L'établissement encore incomplet des fonctions d'assimilation et de desassimilation, chez les tout jeunes animaux, prédispose aux troubles digestifs générateurs des états de dénutrition.

Les facteurs de ces troubles nutritifs sont théoriquement les mêmes chez l'enfant et les jeunes animaux. Ces troubles apparaissent surtout 